\title{
Enfermedades infecciosas y migración. Una responsabilidad compartida.
}

En la actualidad, se estima que más de 244 millones de personas viven fuera de sus países de origen ya sea por conflictos políticos, pobreza, búsqueda de nuevas oportunidades laborales o en educación, la mayoría de los cuales se mueve intrarregionalmente.

En Chile, el fenómeno migratorio se ha incrementado en forma significativa desde 1995, habiéndose cuadriplicado la inmigración latinoamericana. A fines del año 2015 había 465.319 migrantes permanentes residiendo en nuestro país, correspondiente al $2,7 \%$ del total de la población nacional (Ministerio de Desarrollo Social \& Subsecretaría de Evaluación Social, 2015) cifra aún por debajo del promedio mundial de 3,2\% y del de países desarrollados, estimado en $11,3 \%$ de la población según la ONU. De este modo, Chile ocupa el quinto lugar en términos de porcentaje de migrantes con respecto a otros países de Latinoamérica. Los principales países de origen para la migración a Chile son Perú, Argentina, Bolivia, Colombia y Ecuador, y -a partir del 2014- ha habido un aumento importante de población procedente de Haití y Venezuela (Rojas \& Silva 2016).

Especial atención genera el riesgo en salud pública que pudiese implicar la presencia de población migrante en el país de acogida, generándose con frecuencia información alarmista y poco fundada respecto a riesgos de transmisión de enfermedades hacia los habitantes locales, transformándose en foco de discriminación, vulneración de privacidad e incluso violencia. Es por lo tanto fundamental evidenciar la condición de vulnerabilidad que tienen muchos migrantes, y enfatizar que no es la condición de migración en sí la que confiere riesgos de enfermedades, sino más bien su asociación, en el caso chileno, con la situación de pobreza y con el escaso uso de la atención en los servicios de salud (Ministerio de Desarrollo Social \& Subsecretaría de Evaluación Social, 2015; Cabiese et al., 2012).

Son pocas las enfermedades infecciosas transmisibles que efectivamente pudiesen poner en riesgo la población que los acoge, ya que esta tiene generalmente mejores condiciones sanitarias y nutricionales que la hace mucho menos vulnerable a ellas.
En la actualidad, los esfuerzos internacionales de screening de enfermedades transmisibles se concentran fundamentalmente en la tuberculosis (TB) y la mayor parte de los países desarrollados tienen montadas diferentes estrategias de pesquisa de esta (radiografías, estudio de expectoración y test de TB latente) para aquellos migrantes y refugiados que provienen de países de alta endemia (Kunst et al., 2017). En general, estos chequeos médicos se solicitan antes del ingreso (ej: ante solicitud de visa) o bien tras la llegada al país, muchas veces acompañadas de otras restricciones y políticas de inmigración específicas. Sin embargo, la línea entre la protección de la población que acoge y los derechos del migrante es delgada y los screening de salud obligatorios han sido criticados por organismos expertos en derechos humanos que consideran que confieren estigmatización.

En Chile, la tasa de incidencia de TB en todas sus formas fue de 14 por 100.000 habitantes el año 2016, en aumento con respecto a su punto más bajo de 13,5 el año 2014. Las tasas son particularmente elevadas en las regiones del norte de Chile, I y XV Región, con 40 y 26,7 casos por cada 100.000 habitantes respectivamente, coincidiendo con prevalencias altas en los países limítrofes. Entre los factores de riesgo más importantes asociados al diagnóstico de TB estuvo la condición de ser extranjero, correspondiendo al $12,5 \%$ de los casos, en aumento con respecto a años anteriores. De los 16 casos de TB multi-resistente (MDR), el 50\% correspondió a población migrante (MINSAL, 2017). En países más desarrollados como USA, la tasa global de TB ha llegado a ser tan baja como $<3$ por 100.000 habitantes, pero la proporción de los casos que corresponden a migrantes es también elevada (68\% en el año 2016) y las estrategias de pesquisa y prevención se centran fuertemente en esta población de riesgo (Schmit et al., 2017). Considerando la situación epidemiológica nacional, nos parece pertinente y responsable promover también la realización en Chile de un screening básico de TB en aquellos migrantes que provienen de países con alta endemia y que solicitan residencia, con el fin tanto de ofrecerles un tratamiento oportuno como de evitar contagios por diagnósticos tardíos, beneficiándose así todas las partes. 
Otras infecciones, como aquellas de transmisión sexual, son transversales a todos los países y culturas, existiendo en algunos países de América Latina prevalencias elevadas para algunas de ellas, por ejemplo, en Chile, la prevalencia de hepatitis B crónica se estima en alrededor de $0,4 \%$ en la población general, en Perú y Colombia la prevalencia estimada es superior al $2 \%$ y en Haití de hasta $13 \%$. (Schweitzer et al., 2015). En lo relativo a VIH, entre el año 2008 y 2012, 259 extranjeros fueron notificados como positivos en Chile, representando un $2 \%$ de todos los casos a nivel nacional (MINSAL, 2015). Si bien el número de casos de migrantes notificados ha ido en aumento, las crecientes tasas de VIH/SIDA en el país están en la actualidad mayoritariamente representadas por personas chilenas. Es importante considerar que la terapia antiretroviral pese a ser de alto costo es en Chile de acceso universal y no excluye a la población migrante.

Por otra parte, al integrarse al país una población nueva proveniente de diferentes climas y latitudes, nos encontramos con el desafío de tener que aprender a diagnosticar y tratar un grupo de enfermedades infecciosas tropicales hasta ahora ausentes o muy raras en Chile, por ejemplo, la oncocercosis, tracoma, filariasis, lepra, esquistosomiasis, leishmaniasis, histoplasmosis, coccidiomicosis y malaria, entre otras. En Chile, los programas de formación médica general escasamente las mencionan y por lo tanto la sospecha clínica ante estos casos es habitualmente tardía, con métodos diagnósticos y confirmatorios poco accesibles y tratamientos no disponibles en centros habituales, en desmedro de la salud de los pacientes. Cabe destacar que la mayoría de este grupo de enfermedades mencionadas -salvo excepciones- no son transmisibles en nuestro país dado la ausencia del vector o de condiciones ambientales necesarias. Sin embargo, no tratadas a tiempo podrían llegar a ser muy graves o afectar ostensiblemente la calidad de vida del paciente.

En 1993, Chile firmó la Convención Internacional para la Protección de todos los Trabajadores Migratorios y de sus familiares, adoptada por la Asamblea General de las Naciones Unidas, ratificando su postura en el año 2005. En el Instructivo presidencial sobre Política Nacional Migratoria de septiembre del 2008, Chile se definió como un país de acogida, buscando una recepción no discriminatoria, en acuerdo a las convenciones y tratados internacionales vigentes, afirmando que no se podrá denegar prestaciones de salud a los extranjeros que lo requieran. El Ministerio de Salud estableció, el 2009, que los migrantes que tienen visa en trámite pueden obtener carné del Fondo Nacional de Salud (FONASA) que les permita acceder a las atenciones de salud en el sistema público. De este modo, en Chile la población migrante tiene acceso a la salud pública sin discriminación con respecto a la población nativa. Sin embargo, la proporción de migrantes que hace uso del sistema de salud disminuye en directa relación con su condición socioeconómica, nivel educacional y estatus contractual (Cabiese et al., 2012). Se estima que en ellos existe importante desinformación y temor para acceder a los beneficios y prestaciones de salud que confiere el sistema público, probablemente acentuado por barreras lingüísticas y culturales. Además, la condición de permanencia en el país de manera irregular probablemente les aleja aún más de acudir libremente a los servicios de salud en caso de que lo requieran (Cabiese et al., 2012). Por lo tanto, con el fin de prevenir enfermedades transmisibles que pudiesen afectar la salud pública del país y a la vez permitir una atención de salud digna de la población migrante, nos parece fundamental buscar formas de educación y difusión a esta población acerca del acceso a salud, desde el momento en que comienza su proceso de migración, evitando de este modo consultas de morbilidad tardías.

Es de especial preocupación el grupo de mujeres jóvenes, en plena edad reproductiva (se estima que el 52\% de los migrantes en Chile son mujeres) ya que es conocido, a través de estudios hechos en otros países, que frecuentemente las mujeres migrantes buscan apoyo médico en estados avanzados de gravidez siendo la mortalidad materna mayor en ellas en comparación a las mujeres nativas (Ministerio de Desarrollo Social \& Subsecretaría de Evaluación Social, 2015; Rojas \& Silva 2016; Cortés, 2005). Esto Ilama a hacer una alerta en salud materno fetal y en particular con respecto a enfermedades transmisibles al recién nacido, muchas de ellas con prevalencias mayores en sus regiones de origen en enfermedades como VIH, Hepatitis B, HTLV1 y ciertas parasitosis, para las cuales un diagnóstico precoz y tratamiento oportuno son fundamentales para el pronóstico de la madre y para prevenir su transmisión al recién nacido. Por último, a diferencia de Chile donde el programa nacional de inmunizaciones (PNI) alcanza coberturas altas superiores al $90 \%$ desde los años ' 80 , otros países latinoamericanos han tenido históricamente coberturas menores en vacunas, y muy probablemente parte de la población adulta migrante sea aún susceptible a infecciones inmunoprevenibles, y por tanto foco de posibles brotes de infecciones transmisibles como el sarampión o la rubeola.

Qué duda cabe que la migración constituye un trance social complejo que moviliza a las personas hacia lo que esperan será un futuro más promisorio, dejando atrás condiciones adversas, a veces indignas. Parte de las responsabilidades políticas y humanas de los países receptores por tener mayor nivel de desarrollo- es acogerlos y acercarlos a una atención de salud de calidad. Finalmente, considerando la posibilidad de ingreso al país de enfermedades poco conocidas por la mayoría de los 
profesionales médicos nacionales, sería aconsejable disponer de un centro de referencia público de enfermedades del migrante y el viajero, con apoyo clínico (presencial o virtual), laboratorio diagnóstico, y acceso a medicamentos adecuados y oportunos que pudiese resolver mejor aquellos casos de infecciones relacionadas con la globalización.

Dra. Yasna Alarcón V.

Infectología. Hospital Dr. Sótero del Río, Hospital Del

Trabajador. Miembro del Comité de Enfermedades

Emergentes de la Sociedad Chilena de Infectología (SOCHINF)

Dra. María Elvira Balcells.

MSc, DTM\&IH. Profesor Asociado. Departamento de

Enfermedades Infecciosas del Adulto. Pontificia Universidad

Católica de Chile.

\section{Referencias}

Cabiese B, Tunstall H, Pickett K \& Gideon J. (2012). Understanding differences in access and use of healthcare between international immigrants to Chile and the Chilean-born: a repeated cross-sectional population-based study in Chile. Int J Equity Health 11:68.

Cortés, P. (2005).Mujeres migrantes de América Latina y el Caribe: derechos humanos, mitos y duras realidades (CEPAL). Retrieved from http://repositorio.cepal.org/bitstream/handle/11362/7200/1/ S05933_es.pdf

Kunst, H., Burman, M., Arnesen, T. M., Fiebig, L., Hergens, M.-P., Kalkouni, O., de Vries, G. (2017). Tuberculosis and latent tuberculous infection screening of migrants in Europe: comparative analysis of policies, surveillance systems and results. The International Journal of Tuberculosis and Lung Disease, 21(8), 840-851.

Ministerio de Desarrollo Social, \& Subsecretaría de Evaluación Social.CASEM (2015). Encuesta de Caracterización Socioeconómica Nacional. Santiago, Chile. Retrieved from http://observatorio. ministeriodesarrollosocial.gob.cl/casen-multidimensional/casen/ docs/CASEN_2015_Ampliando_la_mirada_sobre_la_pobreza_desigualdad.pdf
MINSAL (2015). Informe Nacional: Evolución de infección VIH/SIDA Chile 1984-2012. Rev. Chil. Infect. 32 (supl. 1) 17-43.

MINSAL (2017). Tuberculosis Informe de situación Chile: 2016. Programa Nacional de Eliminación y Control de la Tuberculosis. Departamento de Enfermedades Transmisibles. División de Prevención y Control de Enfermedades. Ministerio de Salud.

Rojas N \& Silva C. (2016). La migración en chile: Breve reporte y caracterización (OBIMID) (2016). Retrieved from http://www.extranjeria.gob.cl/media/2016/08/informe_julio_agosto_2016.pdf

Schmit, K. M., Wansaula, Z., Pratt, R., Price, S. F., \& Langer, A. J. (2017). Tuberculosis-United States, MMWR (2016). Morbidity and Mortality Weekly Report, 66 (11) 289-294.

Schweitzer, A., Horn, J., Mikolajczyk, R. T., Krause, G., \& Ott, J. J. (2015). Estimations of worldwide prevalence of chronic hepatitis $B$ virus infection: a systematic review of data published between 1965 and 2013. The Lancet, 386(10003), 1546-1555. 